\title{
Etv5 is not required for Schwann cell development but is required to regulate the Schwann cell response to peripheral nerve injury
}

\author{
Abbreviated Title: Etv5 inhibits peripheral nerve injury response \\ Authors: Anjali Balakrishnan ${ }^{1,2}$, Lauren Belfiore ${ }^{1,3}$, Lakshmy Vasan ${ }^{1,3}$, Yacine Touahri ${ }^{1}$, \\ Morgan Stykel $^{4}$, Taylor Fleming ${ }^{1}$, Rajiv Midha ${ }^{5}$, Jeff Biernaskie ${ }^{4}$, Carol Schuurmans ${ }^{1,2,3}$ \\ Institutional Affiliations: \\ ${ }^{1}$ Sunnybrook Research Institute, Toronto, ON, Canada M4N 3M5 \\ ${ }^{2}$ Department of Biochemistry, University of Toronto, Toronto, ON, Canada M5S 1A8 \\ ${ }^{3}$ Department of Laboratory Medicine and Pathobiology, University of Toronto, Toronto, ON, \\ Canada M5S 1A1 \\ ${ }^{4}$ Department of Comparative Biology and Experimental Medicine, University of Calgary, \\ Calgary, AB, Canada T2N 4N1 \\ ${ }^{5}$ Department of Clinical Neurosciences, University of Calgary, Calgary, AB, Canada T2N \\ $4 \mathrm{~N} 1$ \\ Author Contributions: A.B., C.S Designed research; A.B., L.B., L.V., Y.T., M.S., T.F \\ Performed research; A.B., L.B., L.V., Y.T Analyzed data; A.B, L.B., L.V., Y.T., M.S., R.M., \\ J.B., and C.S Wrote the paper
}

\section{Corresponding author: Carol Schuurmans \\ Sunnybrook Research Institute, S116 \\ 2075 Bayview Ave \\ Toronto, ON, Canada \\ M4N 3M5 \\ Phone 416-480-6100 ext 3266 \\ cschuurm@sri.utoronto.ca}

Number of Figures: 5

Number of Words: abstract (226), significance statement (115), introduction (713), discussion (909)

Acknowledgments: C.S. holds the Dixon Family Chair in Ophthalmology Research at the Sunnybrook Research Institute. L.B. is funded by a CIHR Fredrick Banting and Charles Best graduate award-Master's Program.

Conflicts of interest: The authors report no conflict of interest.

Funding sources: This research was funded by a Canadian Institutes of Health Research (CIHR) Project Grant (PJT- 156366) and Alberta Innovates - Health Solutions (AI-HS) Collaborative Research and Innovation Opportunities (CRIO) Project grant (\#201200859) to R.M, J.B. and C.S. 


\section{ABSTRACT}

46 Schwann cells are the principal glial cells of the peripheral nervous system, and their

47 development into myelinating glia is critically dependent on MEK/ERK signaling. Ets-domain

48 transcription factors (Etv1, Etv4, Etv5) are common downstream effectors of MEK/ERK

49 signalling, but so far, only Etvl has been ascribed a role in Schwann cell development, and

50 only in non-myelinating cells. Here, we examined the role of Etv5, which is expressed in

51 Schwann cell precursors, including neural crest cells and satellite glia, in Schwann cell lineage

52 development. We analysed $E t v 5^{t m 1 K m m}$ mutants (designated $E t v 5^{---}$) at embryonic days (E) 12.5,

53 E15.5 and E18.5, focusing on dorsal root ganglia. At these embryonic stages, satellite glia

54 (glutamine synthetase) and Schwann cell markers, including transcriptional regulators (Sox10,

55 Sox9, Tfap2a, Pou3f1) and non-transcription factors (Ngfr, BFABP, GFAP), were expressed

56 in the DRG of wild-type and Etv $5^{-/-}$embryos. Furthermore, by E18.5, quantification of Sox $10^{+}$

57 Schwann cells and $\mathrm{NeuN}^{+}$neurons revealed that these cells were present in normal numbers in

58 the $E t v 5^{-/}$dorsal root ganglia. We next performed peripheral nerve injuries at postnatal day 21,

59 revealing that $E t v 5^{-/-}$mice had an enhanced injury response, generating more Sox $10^{+}$Schwann

60 cells compared to wild-type animals at five days post-injury. Thus, while Etv 5 is not required

61 for Schwann cell development, possibly due to genetic redundancy with Etv1 and/or Etv4, Etv5

62 is an essential negative regulator of the peripheral nerve injury repair response.

\section{SIGNIFICANCE STATEMENT}

66 Our study sought to determine whether the ets domain transcription factor, Etv5, plays a role

67 in regulating Schwann cell development and nerve repair. By using an embryonically and

68 postnatally viable hypomorphic Etv5 mutant allele, we demonstrated that Etv 5 is not required

69 for the development of Schwann cells or other neural crest derivatives in the dorsal root ganglia, 
70 including satellite glia and neurons. Surprisingly, loss of Etv5 had a direct impact on the

71 Schwann cell repair response post-injury, resulting in more Schwann cells populating the distal

72 injured nerve site compared to wild-type animals. Thus, this work describes for the first time a

73 role for Etv5 in regulating the Schwann cell repair response after peripheral nerve injury. 


\section{INTRODUCTION}

76

77
Macroglial cells in the peripheral nervous system (PNS) include Schwann cells and satellite glial cells, which populate peripheral ganglia, including the dorsal root ganglia (DRG) that flank the developing spinal cord. Schwann cells myelinate peripheral nerves, but also wrap non-myelinated axons to regulate neuronal survival and axonal diameter (Jessen and Mirsky, 2005). Conversely, satellite glia encircle peripheral neuronal cell bodies, and have been likened to central nervous system (CNS) astrocytes (Kastriti and Adameyko, 2017). There is also growing support for a role for satellite glia in regulating pain (Jasmin et al., 2010; Ji et al., 2013; Song et al., 2018; Wang et al., 2019; Zhang et al., 2020). Despite their distinct positioning, Schwann cells (wrapping axons) and satellite glia (wrapping neuronal cell bodies) share an embryonic origin, both arising from neural crest cell (NCC) progenitors (Jessen and Mirsky, 2019a). Moreover, satellite glia can transition to a Schwann cell fate, at least in vitro (Cameron-Curry et al., 1993; Hagedorn et al., 2000), highlighting their close lineage relationship, and suggesting that satellite glia could replenish Schwann cells when required for repair.

Multiple signaling molecules and transcriptional regulators regulate Schwann cell development (Castelnovo et al., 2017). The ErbB family of receptor tyrosine kinases (RTKs), which are activated by Neuregulin 1 (NRG1), an EGF family ligand, have emerged as central regulators of Schwann cell precursor (SCP) proliferation, migration and myelination (Newbern and Birchmeier, 2010). In addition, the MEK-ERK signal transduction cascade, activated downstream of RTK signaling, is essential for Schwann cell differentiation, as revealed by the analysis of genetic mutants of ERK1 and ERK2 (Newbern et al., 2011). Conversely, if ERK signaling is ectopically activated in Schwann cells, myelination ensues (Ishii et al., 2013; Sheean et al., 2014). 
ERK kinases have several downstream effectors that they regulate by phosphorylation, including multiple transcription factors (Tsang and Dawid, 2004), such as those of the Etsdomain (helix-turn-helix super family) family (Yang et al., 2013). In Drosophila, Pointed (Pnt) is an ets-domain transcriptional activator that is activated downstream of RTK signaling and which is required for glial cell fate specification (Klaes et al., 1994). In vertebrates, critical ets domain factors activated downstream of RTK signaling include Etv1 (ER81), Etv4 (Pea3) and Etv5 (Erm). Activation of MEK-ERK signaling initiates the expression of Etv1 and Etv5 to specify an oligodendrocyte fate, which are myelinating glial cells in the CNS (Li et al., 2012; Wang et al., 2012; Li et al., 2014; Ahmad et al., 2019). Etv1 is also expressed in Schwann cells (Srinivasan et al., 2007), but it is not required for the development of myelinating Schwann cells (Fleming et al., 2016). Instead, Etv1 facilitates interactions between peripheral axons and non-myelinating Schwann cells in Pacinian corpuscles (Sedy et al., 2006; Fleming et al., 2016).

NCCs and persisting until E12.5 in SCPs and in satellite glial cells (Hagedorn et al., 2000;

Balakrishnan et al., 2016). Etv5 expression is not glial specific, as it is also expressed in TrkA ${ }^{+}$ fate specification but does not affect glial differentiation, NCC survival or proliferation

117 (Paratore et al., 2002). However, whether Etv5 is required for Schwann cell development in vivo has not been addressed.

Strikingly, several genes expressed during Schwann cells development are also expressed post peripheral nerve injury and support a proliferative repair Schwann cell phenotype (Balakrishnan et al., 2016). Here, we analysed Etv $5^{\operatorname{tm} I K m m}$ mutant mice carrying a 122 deletion of exons 2-5 (hereafter designated $\left.E t v 5^{--}\right)($Chen et al., 2005) to ask whether Etv5 is 123 required for Schwann cell development and the peripheral nerve injury response. We found no 
124 evidence for Schwann cell developmental defects in Etv $5^{-/-}$embryos. However, we did observe 125 an expansion of Sox $10^{+}$Schwann cells in Etv $5^{-/-}$peripheral nerves post-injury. Thus, Etv5 acts 126 as a negative regulator of the Schwann cell repair response, and in its absence, more Schwann 127 cells are generated. We discuss our findings in the context of important caveats, such as the 128 potential for genetic redundancy with Etvl and/or Etv4, and our use of a hypomorphic mutant allele, which was necessitated by the early embryonic lethality of Etv 5 null mice.

\section{MATERIAL AND METHODS}

Animals and genotyping. Etv $5^{t m l K m m} / \mathrm{J}$ mice (Stock No. 022300) from Jackson Laboratory (ME, United States) were maintained on a 129/SvJ background. Mice were maintained as heterozygotes in a $12 \mathrm{hr}$ light / $12 \mathrm{hr}$ dark cycle. Heterozygous intercrosses were set up to generate homozygous mutant embryos, designated Etv $5^{-/}$. Pregnancy was determined by detection of a vaginal plug, with the morning of plugging designated as embryonic day (E) 0.5 . PCR genotyping was performed with the following primers: wild-type forward primer: TCT GGC TCA CGA TTC TGA AG; mutant forward primer: AAG GTG GCT ACA CAG GCA AG and common reverse primer: CGG AGG TCA AGC TGT TAA GG.

Embryo collection. Embryo trunks or postnatal nerves were dissected in ice-cold phosphatebuffered saline (PBS), and then fixed overnight in 4\% paraformaldehyde (PFA)/PBS. Fixed tissue was washed in PBS, immersed in $20 \%$ sucrose/PBS overnight, and then blocked in Blocked tissue was sectioned on a Leica cm3050s cryostat (Richmond Hill, ON) at $10 \mu \mathrm{m}$ and collected on SuperFrost ${ }^{\mathrm{TM}}$ Plus slides (Thermo Scientific).

Peripheral nerve crush. Peripheral nerve crush injuries were performed on the sciatic nerve 
149 of P21 wild-type and Etv5 $5^{-/-}$mice as previously described (Balakrishnan et al., 2016). Briefly, 150 P21 animals were anesthetized (5\% isofluorane for induction and 2\% for maintenance),

151 hindlimbs were shaved and sterilized, and a small incision was made to expose the sciatic 152 nerve. A crush injury was performed using \#10 forceps for one minute, and then the muscle 153 and skin were sutured back together. Buprenorpine subcutaneous injection of $0.1 \mathrm{~mL}(100 \mu \mathrm{L}$ 154 of $0.03 \mathrm{mg} / \mathrm{mL}$ ) were administered for pain on the day of surgery and for 4 days following, 155 with the nerve harvested on day 5.

157 Immunohistochemistry. Sections were thawed, rinsed in PBS to remove excess O.C.T., permeabilized in PBT (PBS with $0.1 \%$ TritonX), and then blocked in 10\% normal horse serum/PBT for 1 hour. Primary antibodies were then diluted in blocking solution and incubated on sections overnight at $4^{\circ} \mathrm{C}$, followed by three PBT washes. Species-specific secondary antibodies, conjugated to Alexa 488 or Alexa 555, were diluted 1/500 in PBT and applied to sections for 1 hour. Sections were washed three times in PBT and stained with 4',6-diamidino2-phenylindole (DAPI; Santa Cruz Biotechnology) (1:5000 in PBT). Sections were washed three times in PBS and mounted in AquaPolymount (Polysciences). Primary antibodies included: rabbit anti-Etv5 (Abcam ab102010; 1:300), rabbit anti-Tfap2a (Abcam; ab52222; 1:200), goat anti-Oct6 C-20 (Santa Cruz Biotechnology; sc-11661; 1:50), rabbit anti-Sox9

167 (Millipore; AB5535; 1:500), goat anti-Sox10 (Santa Cruz Biotechnology; sc-17343; 1:400), rabbit anti-Sox10 (Abcam; AB227680; 1:200), Gfap (Dako Cytomation; \#Z0334; 1:500) Ngfr (Millipore; \#07-476;1:500); BFABP (Millipore; ABN14; 1:500) and mouse anti-NeuN (Millipore MAB377; 1:200).

172 RNA in situ hybridization. A digoxygenin-labeled Etv5 riboprobe was generated as 173 previously described using a $10 \times$ labelling mix and following the manufacturer's instructions 
174 (Roche) (Li et al., 2014). The probe was hybridized overnight, and washing and staining procedures were followed as previously described (Touahri et al., 2015).

Waltham MA). Image processing and analysis was performed using Image $\mathrm{J}$ software. Three images per wild-type and Etv $5^{-/}$embryo/nerve were assessed. DAPI channel images were converted into 8-bit format and the threshold was set using weighted mean intensity. Images were inverted for binary conversion. In E18.5 sections, the dorsal root ganglionic region was manually selected using free-form selection tool, while in nerve sections the entire area was assessed. The number of $\mathrm{DAPI}^{+}$cells in the selected region were calculated using particle analysis option. The colocalization of DAPI with the green channel (Sox $10^{+} / \mathrm{NeuN}^{+}$cells) was calculated manually.

Statistical analysis. A minimum of three biological replicates were carried out for all assays. test (when comparing two groups) or One-way ANOVA with TUKEY post corrections (when comparing groups of more than two) were used. All data expressed as mean value \pm standard error of the mean (S.E.M.). In all experiments, a $\mathrm{p}$ value $<0.05$ was considered statistically significant, ${ }^{*} \mathrm{p}<0.05, * * \mathrm{p}<0.01, * * * \mathrm{p}<0.001$, and $* * * * \mathrm{p}<0.0001$

\section{RESULTS}

\section{Schwann cell precursors develop normally in E12.5 Etv5 ${ }^{-/}$peripheral ganglia}


199 Schwann cell precursors (SCPs) in the developing DRG flanking the spinal cord (Fig. 1A,B)

200 (Hagedorn et al., 2000; Balakrishnan et al., 2016). We confirmed the expression of Etv5 in

201 SCPs and satellite glia using RNA in situ hybridization (Fig. 1C) and by co-immunolabeling

202 with Sox10 (Fig. 1D), which marks the Schwann cell lineage at all developmental stages

203 (Balakrishnan et al., 2016). To next assess whether Etv5 is required during this early temporal

204 window for Schwann cell lineage development, we examined E12.5 wild-type and Etv $5^{\text {tm } 1 \mathrm{Kmm}}$

205 mutant embryos (Fig. 1B). Notably, we studied Etv $5^{\text {tmlKmm }}$ mutant mice carrying a deletion of

206 exons 2-5 encoding the initiation codon and a transactivation domain (hereafter $E t v 5^{-/}$) because

207 animals homozygous for a null allele (Etv $\left.5^{\text {tm } 1 \text { Hass }}\right)$, which lack the Etv5 DNA binding domain,

208 die by E8.5 (Chen et al., 2005; Kuure et al., 2010), precluding an analysis of Schwann cell

209 lineage development.

210 We first examined the expression of Sox9 and Sox10, two SRY-box family HMG

211 transcription factors. Sox10 is a specific marker of the Schwann cell lineage, but also marks

212 early migrating NCCs and satellite glia throughout the embryonic period and into postnatal

213 stages and is required for development past the immature Schwann cell (iSC) stage (Kuhlbrodt

214 et al., 1998; Finzsch et al., 2010; Balakrishnan et al., 2016). Sox9 is also expressed early on in

215 the Schwann cell lineage, beginning at the NCC stage (Cheung and Briscoe, 2003;

216 Balakrishnan et al., 2016), and it regulates NCC development (Cheung and Briscoe, 2003). In

217 E12.5 wild-type embryos, Sox9 was expressed more widely, marking SCPs coalescing in the

218 developing DRG and ventral root, but also labelling neural progenitors in the neural tube, part

219 of the CNS, as well as other migratory NCC populations migrating over the surface ectoderm,

220 and mesenchymal cells (Fig. 1 E,G). A very similar pattern of Sox 9 expression was observed

221 in E12.5 Etv $5^{-/-}$embryos, including in SCPs in the DRG, ventral root, and the motor root of the

222 spinal nerve (Fig. $1 F, H$ ). In E12.5 sections through the trunk, Sox9 expression overlapped with

223 Sox10 in the DRG and ventral root, but Sox10 expression was much more restricted to the 
224 Schwann cell lineage (Fig. 1E,I). A similar pattern of Sox 10 expression was seen in E12.5 Etv $5^{-}$

225 /- embryos, with Sox $10^{+}$cells restricted to glial cells in the DRG and ventral root (Fig. $1 F, J$ ). protein (BFABP) is the earliest 'glial' gene turned on in SCPs in a Sox10-dependent manner (Finzsch et al., 2010). Another early marker is Ngfr (also known as p75NTR), a common receptor for neurotrophins, the deletion of which results in a reduced size of peripheral ganglia and reduction in Schwann cell number (von Schack et al., 2001). In E12.5 wild-type embryos, both BFABP (Fig. 2A, $A^{\prime}$ ) and Ngfr (Fig. $2 C, C^{\prime}$ ) were co-expressed with Sox 10 in SCPs in the DRG and also prominently in the dorsal root, the sensory root of the spinal nerve. BFABP also marked the dorsal neural tube and floor plate, while Ngfr was detected more prominently in the ventral neural tube. Very similar patterns of expression were observed in E12.5 Etv $5^{-/-}$ embryos, including prominent expression of BFABP (Fig. 2B, $B^{\prime}$ ) and Ngfr (Fig. 2D, $D^{\prime}$ ) in Sox $10^{+}$SCPs in the DRG and dorsal root.

Thus, at E12.5, there are no notable defects in the general positioning or SCP-specific gene expression in $E t v 5^{-/-}$mutant DRG, ventral, and dorsal roots.

\section{Immature Schwann cells develop normally in E15.5 Etv $5^{-/-}$peripheral ganglia}

Between E12.5 and E15.5, some SCPs persist while others proceed on to form immature Schwann cells (iSCs) that populate the developing spinal ganglia and nerves (Jessen and Mirsky, 2005). We focused on the lumbar spinal cord in E15.5 wild-type and Etv $5^{-/-}$ embryos (Fig. 3A,B), and again examined the expression of Sox9 (Fig. 3C,D) and Sox 10 (Fig. $3 E, F)$. Both Sox 9 and Sox 10 were expressed in a characteristic salt-and-pepper expression

247 pattern in scattered SCPs and iSCs throughout the DRG and in the dorsal and ventral roots in 248 E15.5 wild-type and Etv5 $5^{-/}$embryos (Fig. 3C-F). We also examined the expression of two 
249 additional transcription factors with a later onset of expression in SCPs, including transcription 250 factor AP-2 $\alpha$ (Tfap2a) (Stewart et al., 2001; Balakrishnan et al., 2016) and Sox2, an inhibitor 251 of Schwann cell myelination that is expressed in SCPs and iSCs, but not in pro-myelinating and myelinating Schwann cells (Le et al., 2005; Adameyko et al., 2012; Balakrishnan et al., 2016). Tfap2a was expressed throughout the E15.5 wild-type DRG (Fig. 3G), while Sox2 was only detected in a few Sox $10^{+}$SCPs and iSCs (Fig. 3I,I'), contrasting to the robust expression of Sox2 in the spinal cord ventricular zone (Fig. 3I,I'). Notably, both Tfap2a (Fig. 3H) and Sox2 were similarly expressed in the Schwann cell lineage in E15.5 Etv $5^{-/-}$DRGs (Fig. 3J,J'). Finally, to assess the maturation process of SCPs into iSCs, we examined the expression of non-transcriptional regulators that mark the Schwann cell lineage, including BFABP (Fig. $\left.3 K, L, K^{\prime}, L^{\prime}\right)$, the intermediate filament, glial fibrillary acidic protein (GFAP) (Fig. $3 M, M^{\prime}, N, N^{\prime}$ ) and $\mathrm{Ngfr}$ (Fig. 3O, $O^{\prime}, P, P^{\prime}$ ). All three of these proteins were expressed in scattered Sox $10^{+}$ SCPs and iSCs in both E15.5 wild-type (Fig. $3 K, K^{\prime}, M, M^{\prime}, O, O^{\prime}$ ) and Etv5 $5^{-/}$(Fig. $\left.3 L, L{ }^{\prime}, N, N^{\prime}, P, P^{\prime}\right)$ DRGs. Thus, SCPs and iSCs develop normally in Etv5 hypomorphic mutants.

Late immature Schwann cells/pro-myelinating Schwann cells are detected in E18.5 Etv5${ }^{\text {- }}$ peripheral ganglia and in the dorsal and ventral roots

By E18.5, some iSCs persist, while other iSCs begin to associate with large-diameter axons to become pro-myelinating Schwann cells, whereas iSCs in contact with smaller diameter axons become non-myelinating Schwann cells (Feltri et al., 2015). We first labelled all Schwann cells in the lineage with Sox10 in E18.5 wild-type (Fig. 4A) and Etv5 $5^{-/}$(Fig. 4B) transverse sections through the lumbar spinal cord, and detected similar numbers of Sox10+ Schwann cells in in the DRG, as well as in the dorsal and ventral roots in the wild-type and $272 E t v 5^{-/-}$mutants (Fig. 4I). Further, we examined whether the neuronal cells populating the DRG 273 were affected by labelling the cells with NeuN, a neuronal marker. A similar number of $\mathrm{NeuN}^{+}$ 
274 neurons were observed in both the wild-type and Etv5 ${ }^{-/}$mutant DRGs (Fig. 4C,D,J). Next, we 275 examined the expression of Pou3f1 (Oct-6), which is a marker of late iSCs/pro-myelinating

276 Schwann cells that is required for the transition to a myelinating phenotype transition (Arroyo 277 et al., 1998). We detected Pou3f1 expression in the ventral roots in both E18.5 wild-type (Fig. $2784 E$ ) and $E t v 5^{-/}$(Fig. $4 F$ ) embryos, suggesting that Sox 10 cells mature to a myelinating stage. 279 Finally, to detect satellite glial cells in the developing DRG, we examined the expression of 280 glutamine synthetase (Ohara et al., 2009), revealing that this marker was expressed in both wild-type (Fig. 4G) and Etv5 $5^{-/}$(Fig. 4H) mutant DRGs. Thus, in Etv5 hypomorphic mutants, normal numbers of Schwann cells and DRG neurons are generated during development.

Schwann cells populate the postnatal sciatic nerve in $E t v 5^{--}$mice and respond to injury with an expansion in number

As a final question, we asked whether there were defects in Schwann cells found in the early postnatal nerve, at postnatal day (P) 21, when most pro-myelinating Schwann cells have converted to a myelinating phenotype (Balakrishnan et al., 2016). At P21, we observed expression of Sox10 in scattered cells throughout longitudinal sections of the sciatic nerve in both wild-type (Fig. 5A) and Etv5 $5^{--}$(Fig. 5B) animals, and there were no differences in Schwann cell numbers between these groups in the uninjured nerve (Fig. 5E). We then subjected both P21 wild-type and $E t v 5^{-/}$animals to a sciatic nerve crush injury, which induces a de-differentiation and subsequent expansion of Schwann cells in the distal stump by 5 days post-injury (dpi) (Balakrishnan et al., 2016). We observed an increase in the number of Sox $10^{+}$ distal stumps at $5 \mathrm{dpi}$, suggesting that the repair response occurs normally in $E t v 5^{-/-}$nerves.

297 Interestingly, a significant increase in Sox $10^{+}$cells was observed in Etv5 $5^{--}$injured nerves 
298 compared to wild-type injured nerves ( $\mathrm{p}=0.0027$; Fig. 5E), indicating that Etv5 normally

299 inhibits the ability of Schwann cells to expand post injury.

300 Taken together, these studies suggest that while the Schwann cell lineage develops 301 normally in $E t v 5^{-/}$embryos, from embryonic to early postnatal stages, the response of Etv $5^{-/-}$ 302 Schwann cells to injury is altered, with an increase in Sox $10^{+}$Schwann cells populating near 303 the nerve injury site.

\section{DISCUSSION}

In this study we investigated the role of Etv5 in Schwann cell development using a

307 genetic mutant that deletes exons 2-5, an allele associated with defects in stem cell self-renewal 308 in the spermatogonial lineage (Chen et al., 2005). Using a panel of well annotated Schwann 309 cell markers and quantitative studies at E18.5, we did not observe any notable defects in 310 Schwann cell or neuronal differentiation in Etv5 $5^{-/}$peripheral ganglia at embryonic stages. However, following an acute peripheral nerve injury, which results in the de-differentiation of mature Schwann cells and an increase in Schwann cell proliferation (Jessen and Mirsky, 2019b), more Sox $10^{+}$cells were observed in Etv $5^{-/}$nerves, implicating Etv5 as a negative regulator of the Schwann cell injury response. ERK1/2 signal transduction is activated downstream of NRG1 (Grossmann et al., 2009; Sheean et al., 2014), which is a critical regulator of myelination, and ERK1/2 induce Schwann cell myelination (Ishii et al., 2013; Sheean et al., 2014), including after injury (Harrisingh et al., 2004; Guertin et al., 2005; Napoli et al., 2012;

Kim et al., 2013). Our findings were therefore surprising as our expectation was that Etv5 would be a positive regulator of the injury response.

Notably, in our study we used an Etv5 mutant allele that has a deletion of exons 2-5

$321\left(E t v 5^{t m 1 K m m}\right)$, which results in a very striking reduction in spermatogonial stem cell self-renewal 322 (Chen et al., 2005). In contrast, an Etv5 mutation that removes the DNA binding domain 
323 (Etv $\left.5^{\text {tm } 1 \text { Hass }}\right)$ is embryonic lethal at E8.5, suggesting it is a true null allele (Chen et al., 2005),

324 but precluding us from analysing Schwann cells due to the early embryonic lethality. Due to

325 the embryonic lethality associated with 'more severe' mutant alleles of Etv5, it is believed that

326 our studied Etv5 allele (exon 2-5 knocked out) is hypomorphic rather than a true null allele.

327 Further support for the designation of Etv $5^{\operatorname{tm} 1 \mathrm{Kmm}}$ as a hypomorphic allele comes from the lack

328 of a developmental kidney defect (Lu et al., 2009), whereas the generation of chimeric embryos

329 using Etv $5^{\text {tmlHass }}$ embryonic stem cells revealed that Etv 5 is required for kidney development

330 (Kuure et al., 2010). Future studies on Etv5 function in the Schwann cell lineage would require

331 either the use of such a chimeric approach, or a genetic approach, such as the use of a floxed

332 allele of Etv5 (Zhang et al., 2009). However, even using these alternative approaches, we may

333 not observe a Schwann cell developmental phenotype if there is genetic redundancy. Indeed,

334 different members of the Fgf-synexpression group of ets transcription factors (Etv1, Etv4, Etv5)

335 may compensate for one another to some extent, as shown for Etv4 and Etv5 in kidney

336 development (Kuure et al., 2010). Thus, we may have not uncovered a role for Etv5 in

337 developing Schwann cells due to issues of genetic redundancy. Nevertheless, despite these

338 caveats, we can conclude that the reduced levels of Etv 5 function associated with the Etv $5^{\text {tm } 1 K m m}$

339 allele, which has striking phenotypic consequences in other lineages, does not impact early

340 Schwann cell development, but does impact the Schwann cell response to injury.

341 An important area for future studies will be to further examine the role of Etv 5 in mature

342 Schwann cells. Schwann cell transplants have the potential to aid peripheral nerve repair, and

343 efforts are being made to improve the isolation and expansion of these cells to provide an

344 adequate source for repair purposes. In this regard it is interesting that both human nerve-

345 derived and skin derived Schwann cells cultured in vitro express a large number of Schwann

346 cell markers associated with an early developmental phenotype, which includes Etv5 (Stratton

347 et al., 2017). In our study here we provide the first glimpse that a decline in Etv 5 expression 
348 leads to more number of Schwann cells following a peripheral nerve crush injury. One 349 possibility is that the knockdown of this factor could thus be exploited for regenerative 350 purposes.

RTK-ERK signaling is crucial for Schwann cell differentiation (Newbern et al., 2011) and has been implicated in the myelination process, in part by inducing the expression of promyelinating transcription factors such as $Y y 1$ (He et al., 2010). Other ets-domain transcription 354 factors that are involved in ERK signaling are Etvl and Etv4. While Etv1 is expressed in myelinating Schwann cells (Srinivasan et al., 2007), it is not required for Schwann cell myelination (Fleming et al., 2016). In this regard, it is interesting to note that dominant negative Etv5 misexpression in NCC cultures impacts neuronal fate specification, whereas glial fates are left unperturbed (Paratore et al., 2002). Our study similarly indicated that Etv5 is not required for generation of mature Schwann cells. In contrast, the increase in Sox $10^{+}$cell population in $E t v 5^{-/}$sciatic nerve post-injury (compared to injured wild-type nerves) suggested that loss of Etv5 expression may promote Schwann cell de-differentiation post-injury. Since ERK1/2 signaling is involved in both Schwann cell myelination (Ishii et al., 2013) as well as in promoting a de-differentiated Schwann cell state (Kim et al., 2013), the exact role of Etv5 needs to be further investigated. Questions to be addressed in the future include whether Schwann cells myelinate axons normally in $E t v 5^{-/}$animals, and if Etv5 regulates Schwann cell proliferation post-injury in conjunction with Sox2 and Jun activity, which play an important role in promoting the de-differentiated repair Schwann cell phenotype (Jessen and Mirsky, 2019b). response post-injury. 


\section{References}

374 Adameyko I, Lallemend F, Furlan A, Zinin N, Aranda S, Kitambi SS, Blanchart A, Favaro R, Nicolis S,

375 Lubke M, Muller T, Birchmeier C, Suter U, Zaitoun I, Takahashi Y, Ernfors P (2012) Sox2 and Mitf cross-regulatory interactions consolidate progenitor and melanocyte lineages in the cranial neural crest. Development 139:397-410.

Ahmad ST, Rogers AD, Chen MJ, Dixit R, Adnani L, Frankiw LS, Lawn SO, Blough MD, Alshehri M, Wu W, Marra MA, Robbins SM, Cairncross JG, Schuurmans C, Chan JA (2019) Capicua regulates neural stem cell proliferation and lineage specification through control of Ets factors. Nat Commun 10:2000.

Arroyo EJ, Bermingham JR, Jr., Rosenfeld MG, Scherer SS (1998) Promyelinating Schwann cells express Tst-1/SCIP/Oct-6. J Neurosci 18:7891-7902.

Balakrishnan A, Stykel MG, Touahri Y, Stratton JA, Biernaskie J, Schuurmans C (2016) Temporal Analysis of Gene Expression in the Murine Schwann Cell Lineage and the Acutely Injured Postnatal Nerve. PLoS One 11:e0153256.

Cameron-Curry P, Dulac C, Le Douarin NM (1993) Negative regulation of Schwann cell myelin protein gene expression by the dorsal root ganglionic microenvironment. Eur J Neurosci 5:594-604.

Castelnovo LF, Bonalume V, Melfi S, Ballabio M, Colleoni D, Magnaghi V (2017) Schwann cell development, maturation and regeneration: a focus on classic and emerging intracellular signaling pathways. Neural Regen Res 12:1013-1023.

Chen C, Ouyang W, Grigura V, Zhou Q, Carnes K, Lim H, Zhao GQ, Arber S, Kurpios N, Murphy TL, Cheng AM, Hassell JA, Chandrashekar V, Hofmann MC, Hess RA, Murphy KM (2005) ERM is required for transcriptional control of the spermatogonial stem cell niche. Nature 436:10301034. 
396 Cheung M, Briscoe J (2003) Neural crest development is regulated by the transcription factor Sox9.

397 Development 130:5681-5693.

Chotteau-Lelievre A, Desbiens X, Pelczar H, Defossez PA, de Launoit Y (1997) Differential expression patterns of the PEA3 group transcription factors through murine embryonic development.

Feltri ML, Poitelon Y, Previtali SC (2015) How Schwann Cells Sort Axons: New Concepts.

402 Oncogene 15:937-952.

Finzsch M, Schreiner S, Kichko T, Reeh P, Tamm ER, Bosl MR, Meijer D, Wegner M (2010) Sox10 is required for Schwann cell identity and progression beyond the immature Schwann cell stage.

Fleming MS, Li JJ, Ramos D, Li T, Talmage DA, Abe SI, Arber S, Luo W (2016) A RET-ER81-NRG1

Grossmann KS, Wende H, Paul FE, Cheret C, Garratt AN, Zurborg S, Feinberg K, Besser D, Schulz H,

Guertin AD, Zhang DP, Mak KS, Alberta JA, Kim HA (2005) Microanatomy of axon/glial signaling Peles E, Selbach M, Birchmeier W, Birchmeier C (2009) The tyrosine phosphatase Shp2 (PTPN11) directs Neuregulin-1/ErbB signaling throughout Schwann cell development. Proceedings of the National Academy of Sciences of the United States of America 106:16704-16709. satellite cells by neuregulin signaling. Developmental biology 219:44-58. 
419 Harrisingh MC, Perez-Nadales E, Parkinson DB, Malcolm DS, Mudge AW, Lloyd AC (2004) The

420 Ras/Raf/ERK signalling pathway drives Schwann cell dedifferentiation. The EMBO journal

$421 \quad 23: 3061-3071$.

422 He Y, Kim JY, Dupree J, Tewari A, Melendez-Vasquez C, Svaren J, Casaccia P (2010) Yy1 as a molecular

$423 \quad$ link between neuregulin and transcriptional modulation of peripheral myelination. Nat

$424 \quad$ Neurosci 13:1472-1480.

425

426

427

428

429

430

431
Ishii A, Furusho M, Bansal R (2013) Sustained activation of ERK1/2 MAPK in oligodendrocytes and schwann cells enhances myelin growth and stimulates oligodendrocyte progenitor expansion. J Neurosci 33:175-186.

Jasmin L, Vit JP, Bhargava A, Ohara PT (2010) Can satellite glial cells be therapeutic targets for pain control? Neuron Glia Biol 6:63-71.

Jessen KR, Mirsky R (2005) The origin and development of glial cells in peripheral nerves. Nature reviews Neuroscience 6:671-682.

Jessen KR, Mirsky R (2019a) Schwann Cell Precursors; Multipotent Glial Cells in Embryonic Nerves. Front Mol Neurosci 12:69.

Jessen KR, Mirsky R (2019b) The Success and Failure of the Schwann Cell Response to Nerve Injury. Front Cell Neurosci 13:33.

Ji RR, Berta T, Nedergaard M (2013) Glia and pain: is chronic pain a gliopathy? Pain 154 Suppl 1:S1028.

Kastriti ME, Adameyko I (2017) Specification, plasticity and evolutionary origin of peripheral glial cells. Curr Opin Neurobiol 47:196-202. 
440 Kim HA, Mindos T, Parkinson DB (2013) Plastic fantastic: Schwann cells and repair of the peripheral

441 nervous system. Stem Cells Transl Med 2:553-557.

442 Klaes A, Menne T, Stollewerk A, Scholz H, Klambt C (1994) The Ets transcription factors encoded by

443 the Drosophila gene pointed direct glial cell differentiation in the embryonic CNS. Cell

$444 \quad 78: 149-160$.

Kuhlbrodt K, Herbarth B, Sock E, Hermans-Borgmeyer I, Wegner M (1998) Sox10, a novel transcriptional modulator in glial cells. J Neurosci 18:237-250.

Kuure S, Chi X, Lu B, Costantini F (2010) The transcription factors Etv4 and Etv5 mediate formation of the ureteric bud tip domain during kidney development. Development 137:1975-1979.

Le N, Nagarajan R, Wang JY, Araki T, Schmidt RE, Milbrandt J (2005) Analysis of congenital

Li S, Mattar P, Dixit R, Lawn SO, Wilkinson G, Kinch C, Eisenstat D, Kurrasch DM, Chan JA, Schuurmans C (2014) RAS/ERK signaling controls proneural genetic programs in cortical development and gliomagenesis. J Neurosci 34:2169-2190. 
Napoli I, Noon LA, Ribeiro S, Kerai AP, Parrinello S, Rosenberg LH, Collins MJ, Harrisingh MC, White IJ, Woodhoo A, Lloyd AC (2012) A central role for the ERK-signaling pathway in controlling Schwann cell plasticity and peripheral nerve regeneration in vivo. Neuron 73:729-742.

Newbern J, Birchmeier C (2010) Nrg1/ErbB signaling networks in Schwann cell development and myelination. Seminars in Cell \& Developmental Biology 21:922-928.

Newbern JM, Li X, Shoemaker SE, Zhou J, Zhong J, Wu Y, Bonder D, Hollenback S, Coppola G, Geschwind DH, Landreth GE, Snider WD (2011) Specific functions for ERK/MAPK signaling during PNS development. Neuron 69:91-105.

Ohara PT, Vit JP, Bhargava A, Romero M, Sundberg C, Charles AC, Jasmin L (2009) Gliopathic pain: when satellite glial cells go bad. Neuroscientist 15:450-463.

Paratore C, Brugnoli G, Lee HY, Suter U, Sommer L (2002) The role of the Ets domain transcription factor Erm in modulating differentiation of neural crest stem cells. Developmental biology 250:168-180.

Sedy J, Tseng S, Walro JM, Grim M, Kucera J (2006) ETS transcription factor ER81 is required for the Pacinian corpuscle development. Dev Dyn 235:1081-1089.

Sheean ME, McShane E, Cheret C, Walcher J, Muller T, Wulf-Goldenberg A, Hoelper S, Garratt AN, Kruger M, Rajewsky K, Meijer D, Birchmeier W, Lewin GR, Selbach M, Birchmeier C (2014) Activation of MAPK overrides the termination of myelin growth and replaces Nrg1/ErbB3 signals during Schwann cell development and myelination. Genes Dev 28:290-303.

Song J, Ying Y, Wang W, Liu X, Xu X, Wei X, Ruan X (2018) The role of P2X7R/ERK signaling in dorsal root ganglia satellite glial cells in the development of chronic postsurgical pain induced by skin/muscle incision and retraction (SMIR). Brain Behav Immun 69:180-189. 
484 Srinivasan R, Jang SW, Ward RM, Sachdev S, Ezashi T, Svaren J (2007) Differential regulation of NAB

$485 \quad$ corepressor genes in Schwann cells. BMC Mol Biol 8:117.

Stewart HJ, Brennan A, Rahman M, Zoidl G, Mitchell PJ, Jessen KR, Mirsky R (2001) Developmental regulation and overexpression of the transcription factor AP-2, a potential regulator of the timing of Schwann cell generation. Eur J Neurosci 14:363-372.

Stratton JA, Kumar R, Sinha S, Shah P, Stykel M, Shapira Y, Midha R, Biernaskie J (2017) Purification and Characterization of Schwann Cells from Adult Human Skin and Nerve. eNeuro 4.

Touahri Y, Adnani L, Mattar P, Markham K, Klenin N, Schuurmans C (2015) Non-isotopic RNA In Situ Hybridization on Embryonic Sections. Curr Protoc Neurosci 70:1 22 21-21 2225.

Tsang M, Dawid IB (2004) Promotion and attenuation of FGF signaling through the Ras-MAPK pathway. Sci STKE 2004:pe17.

von Schack D, Casademunt E, Schweigreiter R, Meyer M, Bibel M, Dechant G (2001) Complete ablation of the neurotrophin receptor p75NTR causes defects both in the nervous and the vascular system. Nat Neurosci 4:977-978.

Wang Y, Kim E, Wang X, Novitch BG, Yoshikawa K, Chang LS, Zhu Y (2012) ERK inhibition rescues defects in fate specification of Nf1-deficient neural progenitors and brain abnormalities. Cell $150: 816-830$

Wang Z, Li L, Yang R, Xu X, Liang S (2019) P2X receptors mediated abnormal interaction between satellite glial cells and neurons in visceral pathological changes. Cell Biol Int.

Yang SH, Sharrocks AD, Whitmarsh AJ (2013) MAP kinase signalling cascades and transcriptional regulation. Gene 513:1-13. 
Zhang YY, Song N, Liu F, Lin J, Liu MK, Huang CL, Liao DQ, Zhou C, Wang H, Shen JF (2020) Activation of the RAS/B-RAF-MEK-ERK pathway in satellite glial cells contributes to substance $p$ mediated orofacial pain. Eur J Neurosci 51:2205-2218.

Zhang Z, Verheyden JM, Hassell JA, Sun X (2009) FGF-regulated Etv genes are essential for repressing Shh expression in mouse limb buds. Dev Cell 16:607-613.

\section{FIGURE LEGENDS}

Figure 1. Schwann cell transcriptional regulators are expressed normally in E12.5 Etv $5^{-/-}$ Schwann cell precursors (SCPs). Etv $5^{+}$neural crest cells give rise to Etv $5^{+}$sensory neurons, Etv $5^{+}$satellite glia, and Etv5- Schwann cell precursors (A). Schematic representation of E12.5 trunk section (B). Distribution of Etv5 transcripts in E12.5 transverse sections of the spinal cord (C). Co-expression of Etv5 (red, D), Sox9 (red, E-G,I) and Sox10 (green, D,E,F,H,J) with DAPI counterstain (blue) in E12.5 wild-type (D,E,G,I) and Etv $5^{-/-}(\mathrm{F}, \mathrm{H}, \mathrm{J})$ embryos. Inset in (D) presents 3X magnified image of region marked by a dotted box. dr, dorsal root; drg, dorsal root ganglion; sc, spinal cord; vr, ventral root. Scale bars, $60 \mu \mathrm{m}$.

\section{Figure 2. Schwann cell non-transcription factor lineage markers are expressed normally} in E12.5 Etv5 $^{-/-}$SCPs. Co-expression of Sox10 (green, A-D) with BFABP (red, A,B, black/white, A',B') and Ngfr (red, C,D, black/white, C',D'), counterstained with DAPI (blue, A-D) in E12.5 wild-type (A,A',C,C') and $E t v 5^{-/-}\left(\mathrm{B}, \mathrm{B}^{\prime}, \mathrm{D}, \mathrm{D}^{\prime}\right)$ transverse sections through the trunk. dr, dorsal root; drg, dorsal root ganglion; sc, spinal cord ; vr, ventral root. Scale bars, 60 $\mu \mathrm{m}$.

\section{Figure 3. Schwan cell lineage markers are expressed normally in E15.5 Etv5 ${ }^{-/-}$immature} Schwann cells (iSCs). Low magnification DAPI-stained images of transverse sections through the lumbar spinal cord of E15.5 wild-type (A) and Etv5 $5^{--}$(B) embryos. (C-H) Expression of 
529 Sox9, Sox10 and Tfap2a in E15.5 wild-type (C,E,G) and Etv5 $5^{--}$(D,F,H) transverse sections 530 through the lumbar spinal cord. (I-P) Co-expression of Sox10 (green, I-P) with Sox2 (red, I,J, 531 black/white, I',J'), BFABP (red, K,L, black/white, K',L'), GFAP (red, M,N, black/white, 532 M',N'), and Ngfr (red, O,P, black/white, O',P'), counterstained with DAPI (blue, I-P) in E12.5 533 wild-type (I,I',K,K',M,M',O,O') and Etv5 $5^{-/-}$(J,J',L,L',N,N',P,P') transverse sections through 534 the lumbar spinal cord. dr, dorsal root; drg, dorsal root ganglion; sc, spinal cord; vr, ventral 535 root. Scale bars (A,B), $100 \mu \mathrm{m}$; (C-P'), $60 \mu \mathrm{m}$.

Figure 4. Schwan cell lineage markers are expressed normally in E18.5 Etv5 ${ }^{-/-}$late immature/pro-myelinating Schwann cells. Labelling of Sox10 (A,B), NeuN (C,D), Pou3f1 538 (E,F), and Glutamine Synthetase (GS; G,H) with DAPI counterstain in E18.5 wild-type 539 (A,C,E,G) and Etv $5^{-/-}$(B,D.F.H) transverse sections through the lumbar spinal cord. 540 Quantification of percentage of Sox $10^{+} / \mathrm{DAPI}^{+}$cells (I) in the dorsal root ganglia, dorsal and 541 ventral root, and $\mathrm{NeuN}^{+} / \mathrm{DAPI}^{+}$cells $(\mathrm{J})$ in the dorsal root ganglia. Error bars=s.e.m.. drg, dorsal 542 root ganglion; sc, spinal cord; vr, ventral root; ns, non-significant. Scale bars, (A,B), $60 \mu$ m; 543 (C,D,G.H), $40 \mu \mathrm{m} ;(\mathrm{E}, \mathrm{F}), 20 \mu \mathrm{m}$.

544 Figure 5. Increase in $\operatorname{Sox}^{+} 0^{+}$Schwann cells post-injury in Etv5 $5^{-/-}$sciatic nerve. Sox 10 545 expression in longitudinal sections of the uninjured (A,B) and injured (C,D) P21 sciatic nerve 546 from wild-type $(\mathrm{A}, \mathrm{C})$ and $E t v 5^{-/-}(\mathrm{B}, \mathrm{D})$ animals. Quantification of number of Sox $10^{+}$cells $(\mathrm{E})$ 547 in the entire section. Error bars=s.e.m. *, $\mathrm{p}<0.05 ; * *, \mathrm{p}<0.01 ; * * *, \mathrm{p}<0.005$. Scale bars, 20 $548 \mu \mathrm{m}$. 


\section{A}

bioRxiv preprint doi: https://doi.org/10.1101/2020.09.23.309815; this version-msted September 24, 2020. The copyright holder for this preprint

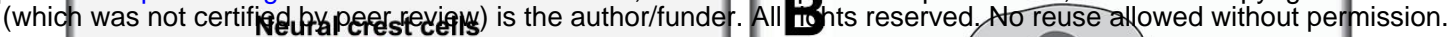
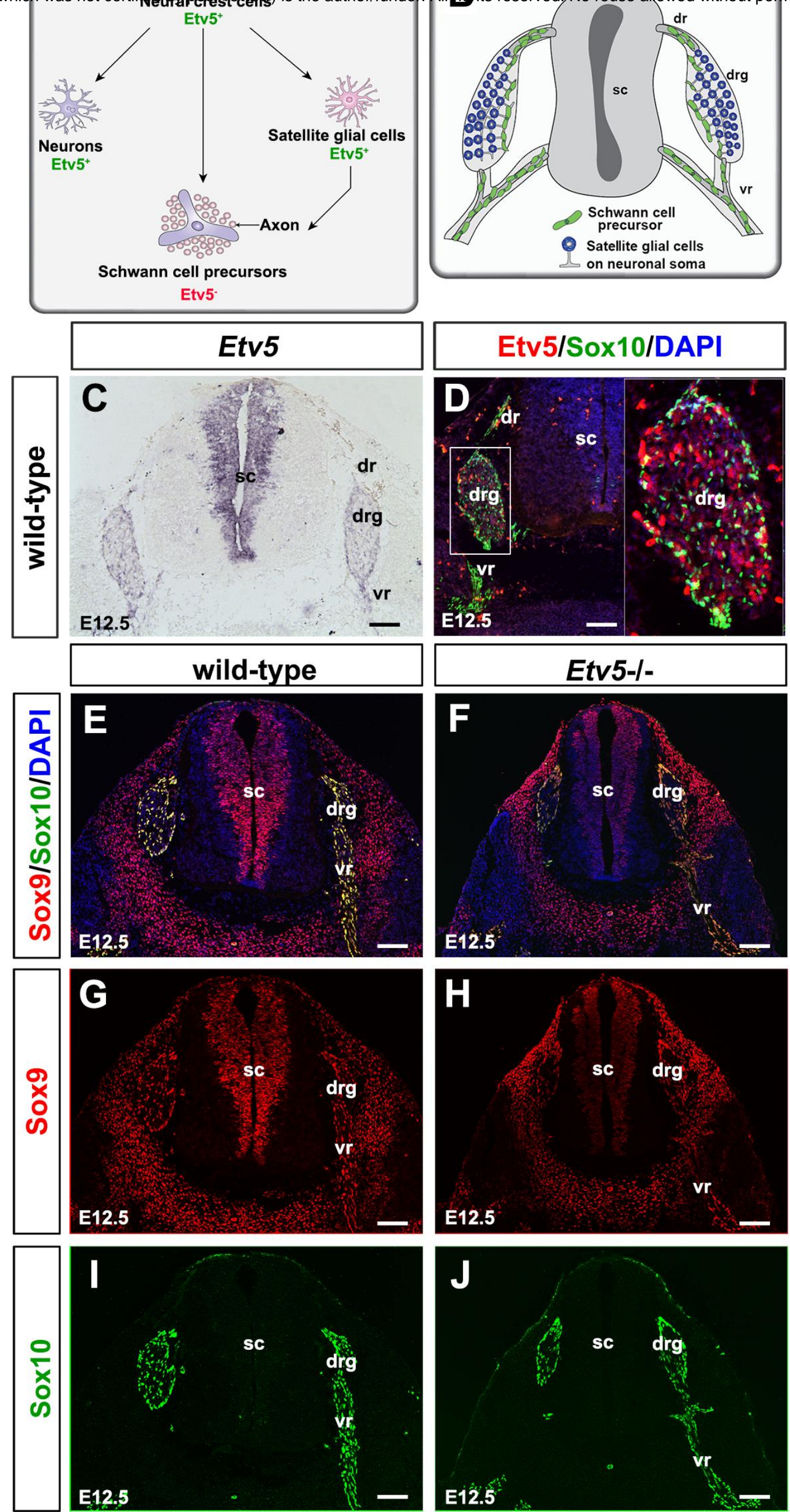

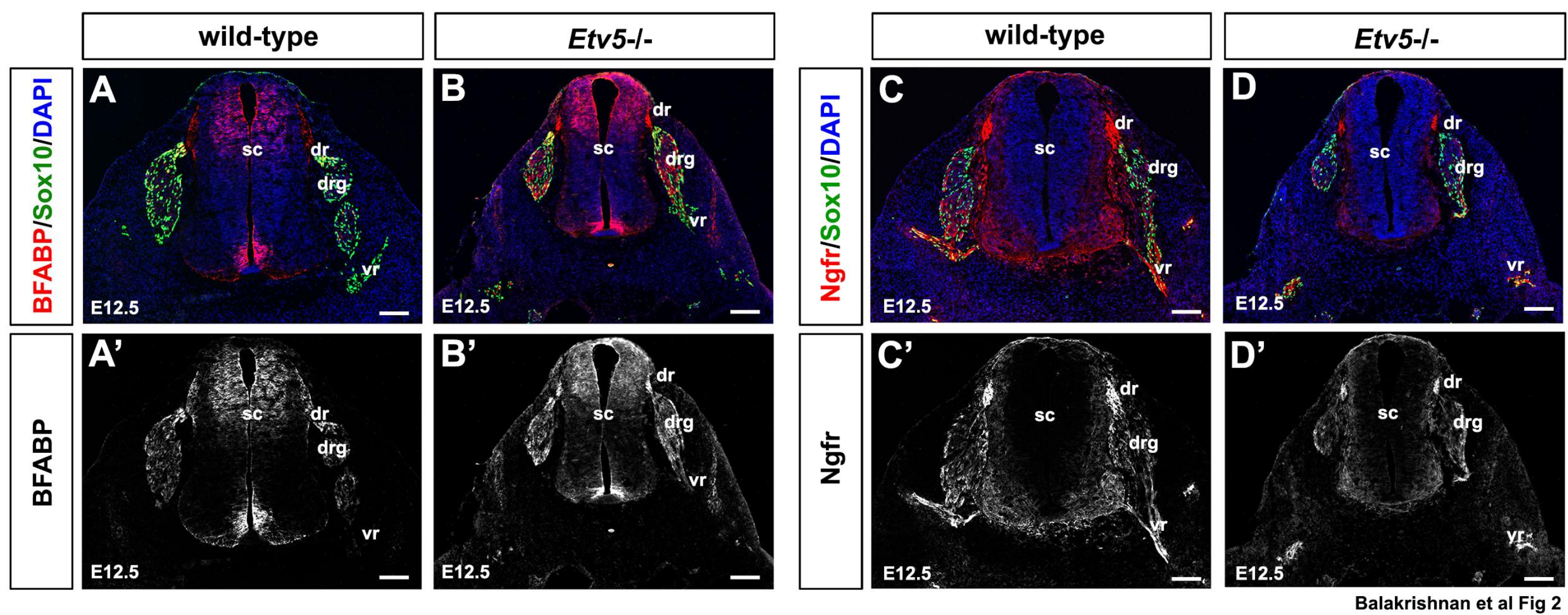


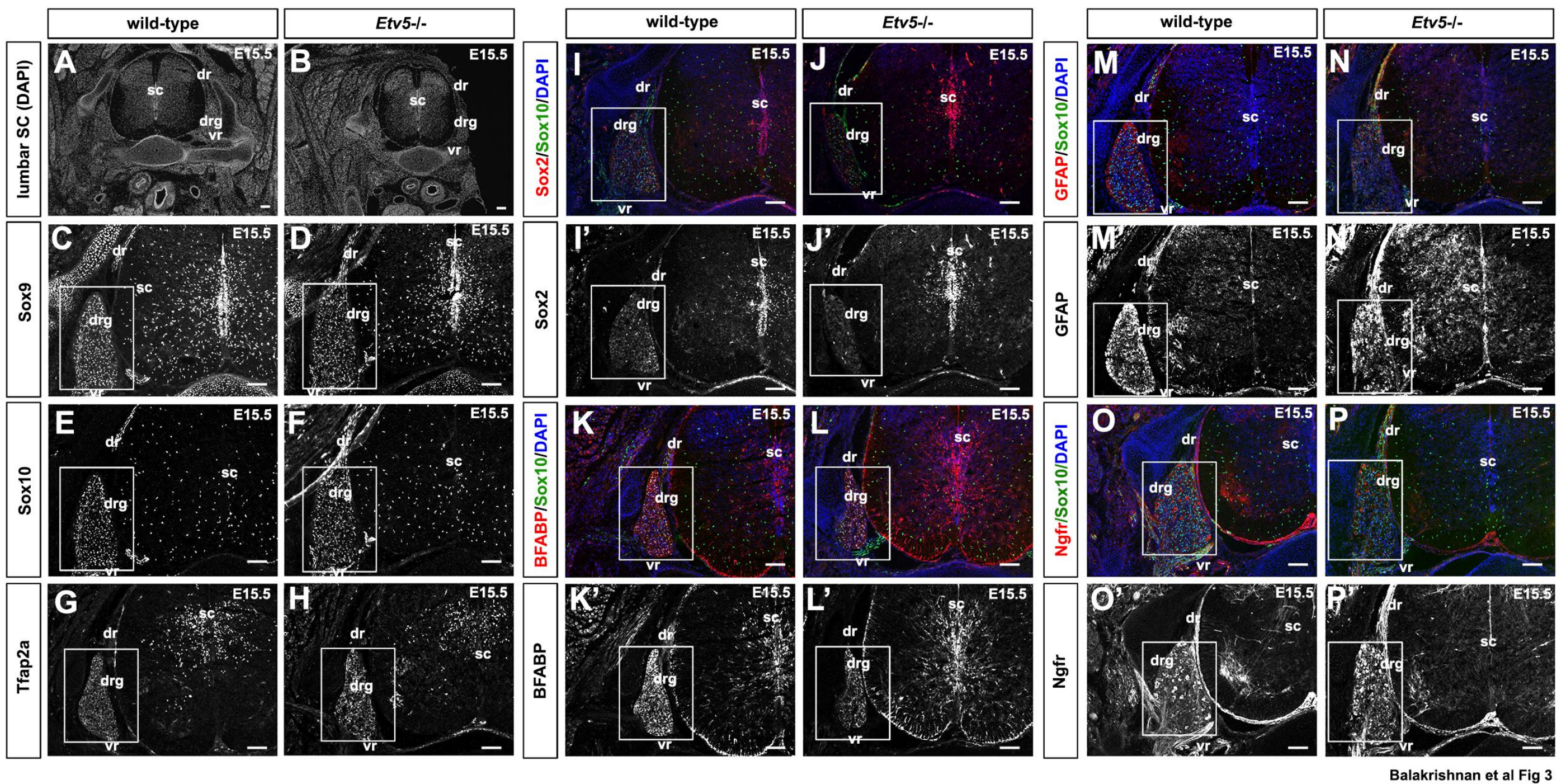



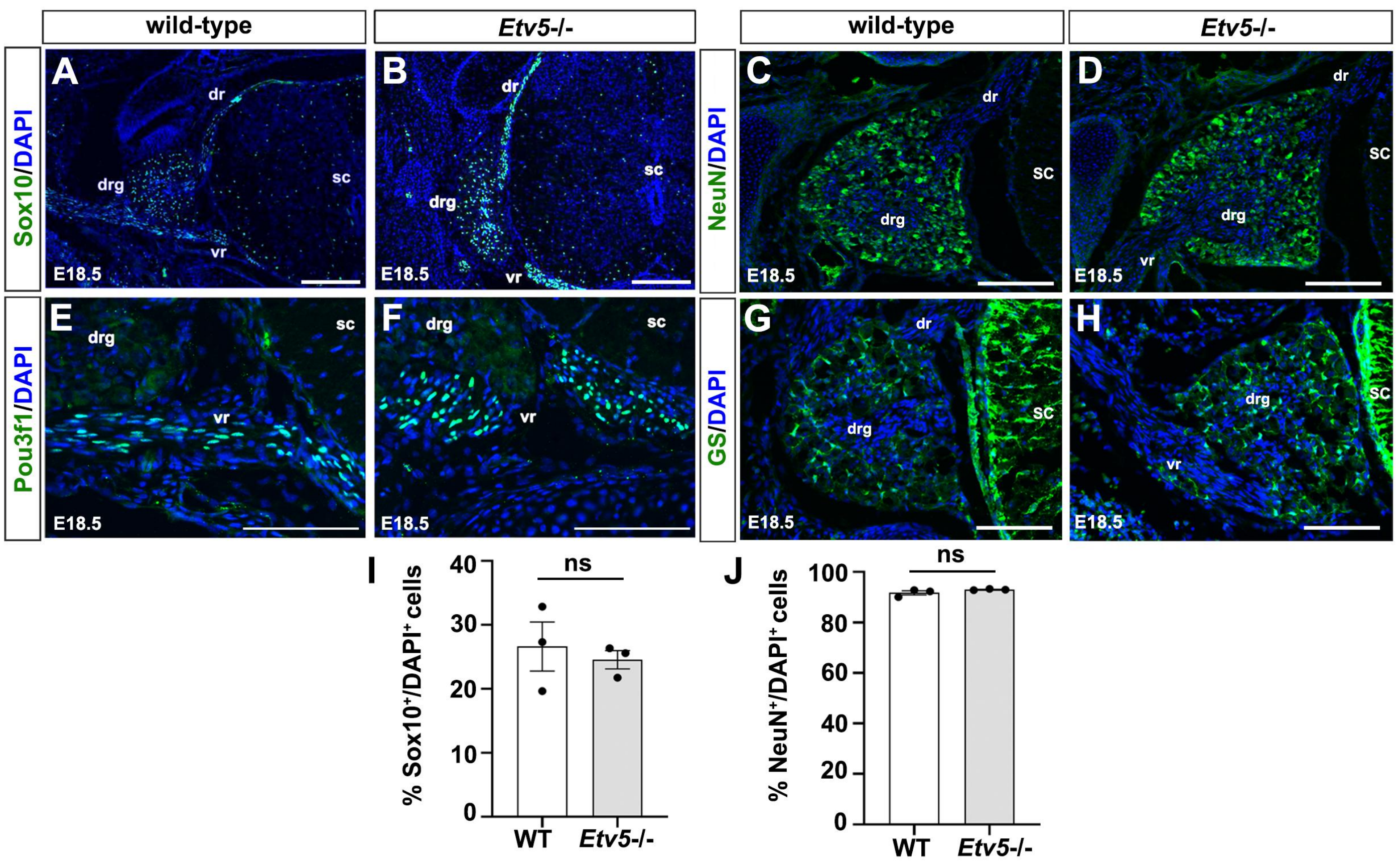

Balakrishnan et al Fig 4 
bioRxiv preprint doi: https://doi.org/10.1101/2020.09.23.309815; this version posted September 24, 2020. The copyright holder for this preprint (which was not certified by peer review) is the author/funder. All rights reserved. No reuse allowed without permission.

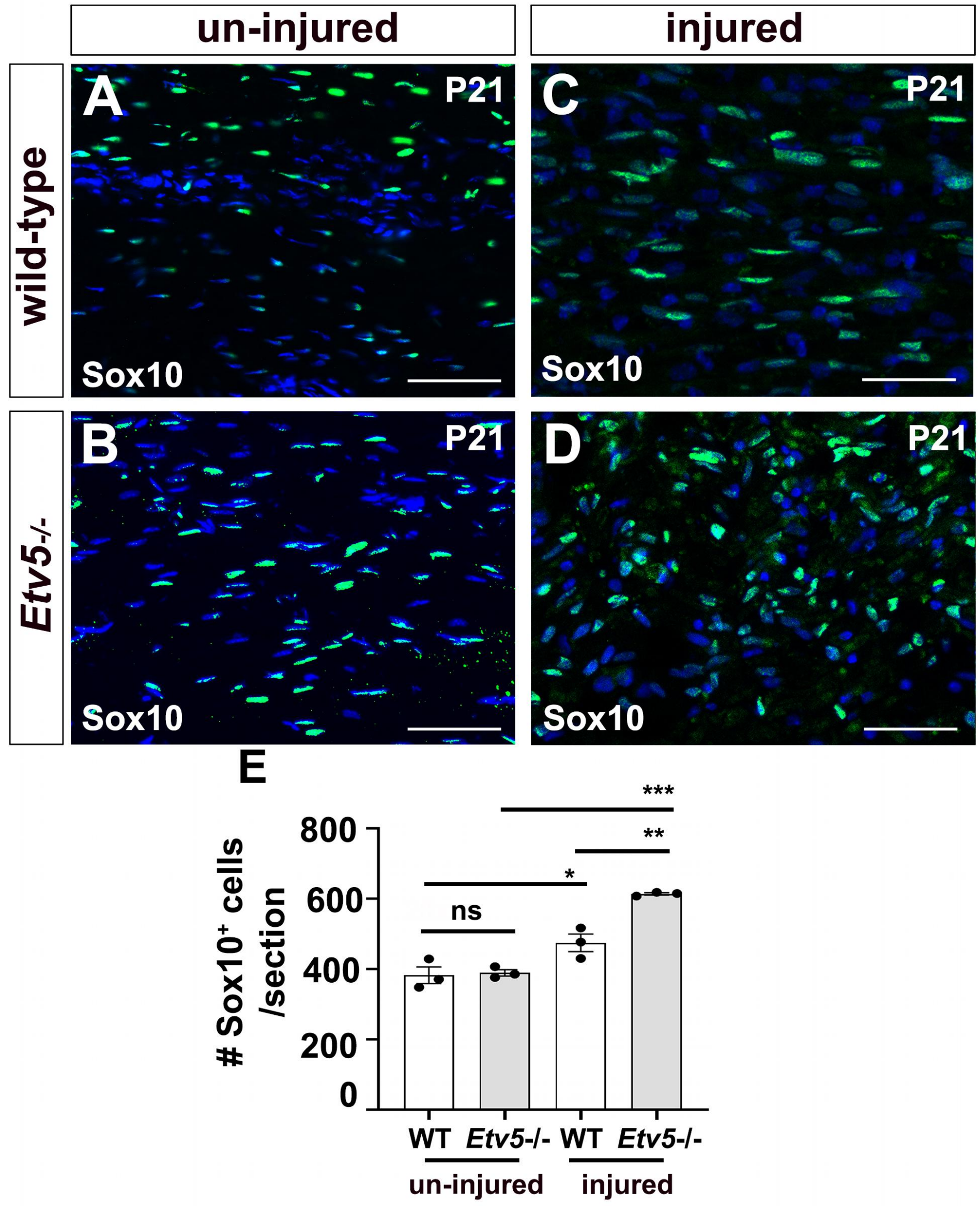

Balakrishnan et al Fig 5 